\title{
SAR Distribution in Microwave Breast Screening: Results with TWTLTLA Wideband Antenna
}

\author{
Adam Santorelli, Milica Popović \\ Department of Electrical and Computer Engineering, McGill University \\ Montreal, Canada \\ adam.santorelli@mail.mcgill.ca \\ milica.popovich@mcgill.ca
}

\begin{abstract}
This paper focuses on safety assessment of microwave breast screening systems. Using numerical analysis, a heterogeneous, life-like breast phantom is illuminated by an antenna, currently used in experimental microwave imaging systems, from several locations and at several frequencies. These frequencies correspond to the content of experimental pulses used in microwave detection systems. The computed specific absorption rate (SAR) values, averaged over $10 \mathrm{~g}$ of tissue, imply that the microwave screening techniques comply with previously established safety limits for the SAR.
\end{abstract}

\section{INTRODUCTION}

Breast cancer remains the most commonly diagnosed cancer worldwide amongst women. The early detection and treatment of these cancers is critical for the successful treatment of the disease [1]. Microwave imaging is a novel technique which aims to provide a complimentary imaging modality to the current standards in breast cancer screening methods. X-ray mammography, the gold standard for early screening, exposes patients to ionizing radiation, suffers from a high occurrence of false-positives, and requires a biopsy for the confirmation of a malignant tumour. Microwave imaging has shown promise as an early-stage breast cancer screening technique which can address some of the known issues with mammography. The presence of small tumours can be detected by exposing the breast tissue to incident microwave energy and recovering the scattered waves [2]. This illumination of the breast with microwaves will cause the breast tissues to absorb some of the incident energy. This absorbed energy will subsequently cause the temperature of the breast to increase. Thus, it is necessary to ensure that this heating remains well below the safety limits, previously set by relevant authorities, to guarantee there is no damage to the illuminated breast tissue [3].

This work presents the results of conducting finitedifference time-domain (FDTD) analysis of the energy levels deposited into the human breast resulting from the exposure of microwave energy emanating from an antenna placed in several specific locations while excited by several frequencies. Previous safety assessments have focused on two-dimensional analysis or relied on tissue properties that were reported prior to the thorough investigation in [4]. Recently, we have conducted a safety assessment using an anatomically realistic breast model, complete with tissue properties based on pixel intensity and correlated with the data in [4]. This analysis, however, was based on the illumination of the breast with a plane wave [5]. The analysis presented in this paper is conducted by placing a three-dimensional (3-D) anatomically realistic breast model in the near-field of an antenna, a scenario which is the commonly employed method in most microwave imaging techniques.

\section{BACKGROUND}

Microwave imaging operates on the premise that tissues within the breast have inherent differences in both electrical conductivity and permittivity. This contrast in electrical properties of malignant and healthy breast tissue causes the non-uniform absorption and scattering of incident microwave energy within the complex heterogeneous breast structure. Relatively low propagation loss at microwave frequencies in the breast tissue allows for partial recovery of the transmitted and reflected microwaves. These recovered signals can then be used for imaging purposes.

In the microwave frequency range, the primary cause of tissue damage from electromagnetic (EM) exposure is due to the heating of the tissue. This heating is caused by the absorption of energy from the incident wave [3]. The SAR parameter is commonly considered by current safety standards to assess the amount of energy deposited in human tissue. Table I contains data in regards to the maximal permissible SAR levels for various situations [3], [6]. The goal of microwave imaging devices for breast cancer screening is to create an easily accessible technology, and as such, its operation may be carried out in public locations. Hence, it is necessary to follow the safety limits associated with an uncontrolled environment. Furthermore, this investigation is concerned with radiation of the breast placed within the nearfield of an antenna. Thus, we are concerned with peak local SAR values averaged over a small quantity of mass [6]. As shown in Table I, the limits for the peak local SAR values can be averaged over the whole-body (WB), $1 \mathrm{~g}$ of tissue (1G), or $10 \mathrm{~g}$ of tissue (10G).

Previous work, as shown in [5], [7], has concluded that the application of microwave imaging modalities represents a safe imaging technique. However, these studies were based on either plane-wave illumination [5], or used a breast model based on outdated tissue properties [7]. The analysis presented within this paper makes use of the recently reported tissue parameters in [4] by implementing the breast model from [8] and exposing the breast model to radiation from a specifically designed antenna. The breast model is placed within the near- 
field of the antenna and the antenna is swept through four possible locations. This analysis will help to conclude whether the radiation pattern of the antenna, as well as the location of the antenna relative to the breast, changes the location and magnitude of peak local SAR values.

TABLE I

SAR EXPOSURE LIMITS:

$\mathrm{WB}=$ WHOLE BODY AVERAGING, $1 \mathrm{G}=$ AVERAGING OVER 1 GRAM OF TISSUE, $10 \mathrm{G}=$ AVERAGING OVER 10 GRAMS OF TISSUE

\begin{tabular}{|c|c|c|}
\hline & $\begin{array}{c}\text { Controlled } \\
\text { Environment }[\mathbf{W} / \mathbf{k g}]\end{array}$ & $\begin{array}{c}\text { Uncontrolled } \\
\text { Environment }[\mathbf{W} / \mathbf{k g}]\end{array}$ \\
\hline $\mathbf{S A R}_{\mathbf{W B}}$ & 0.4 & 0.08 \\
\hline $\mathbf{S A R}_{\mathbf{1 G}}$ & 8 & 1.6 \\
\hline $\mathbf{S A R}_{\mathbf{1 0 G}}$ & 20 & 4 \\
\hline
\end{tabular}

\section{MODELS AND METHODS}

The following section details the requirements needed to set up the entire simulation scenario. A description of the modelling procedure for the antenna, breast model, and the antenna excitation structure is provided. Each sub-section characterizes and details specific parameters about each of these models. The modeling and simulations are carried out in the commercial FDTD solver software SEMCAD-X [9]. The 3-D numerical breast model described in [8], and subsequently used in this analysis, has been tailored to facilitate efficient use of SEMCAD-X.

\section{A. Antenna Characterization}

The antenna used in this report is a Traveling Wave Tapered and Loaded Transmission Line Antenna (TWTLTLA) [10]. It is a broadband antenna specifically designed to operate for the microwave imaging of the breast. Hence its compact design, $0.635 \mathrm{~mm} \times 12 \mathrm{~mm} \times 15.8 \mathrm{~mm}$, and its ideal performance in a background medium with electrical properties similar to that of the fatty breast tissue. Fig. 1 provides an image of the antenna designed in [10]. We provide a reference of the antenna size by comparing it to a Canadian penny. Fig. 2 graphs the antenna performance by reporting the simulated results of the return loss (S11) for the antenna. The performance of the antenna is computed for operation in several backgrounds, in particular air, a fat-mimicking matching medium, and a canola oil matching medium. We note that at frequencies below $10 \mathrm{GHz}$ the antenna performance is best when it is embedded in the fat-mimicking material.

The antenna is placed in one of four specific locations near the breast. The locations of the antenna have been chosen to match the locations of the antenna in the experimental system reported in [11]. By choosing these locations we match the simulation scenario to a microwave imaging system currently under investigation, correlating the results of this safety assessment to a true experimental system. The antenna is arranged in such a fashion to ensure it remains $2.5 \mathrm{~mm}$ away from the skin of the breast as in [11].

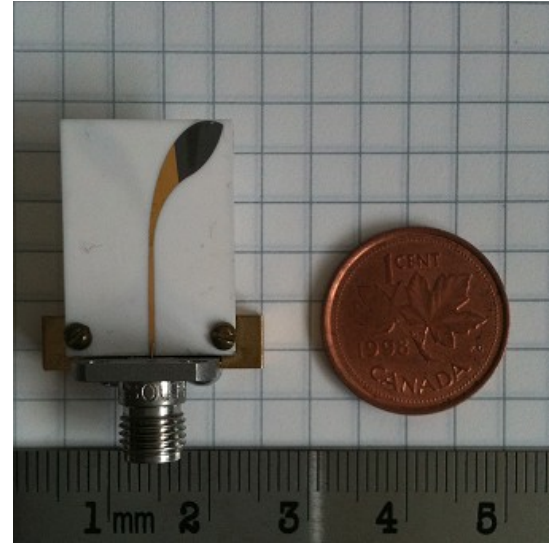

Fig.1. Image of the antenna placed next to a Canadian penny for reference The image is shown to scale, each square of the background corresponds to a $5 \mathrm{~mm} \times 5 \mathrm{~mm}$ area.

\section{B. Breast Model}

The shape and interior geometry of the breast model is derived from MRI images. The pixel intensity of these images allows for tissues to be differentiated, and it is used to map the permittivity and conductivity values to specific tissue areas appropriately [8]. This results in a 3-D profile of electric parameters that realistically represent the heterogeneous nature of the human breast anatomy and can be incorporated in the electromagnetic simulation.

The complete simulation environment consists of the breast model, a skin layer, the chest wall, and a matching medium. The chest wall consists of a $1.5-\mathrm{mm}$ skin layer, a $3-\mathrm{mm}$ fat layer, and a $5-\mathrm{mm}$ muscle layer. The $1.5-\mathrm{mm}$ skin layer extends to cover the entire breast model. This model is embedded in a fat-mimicking matching medium in order to improve the performance of the antenna in the frequency range of interest.

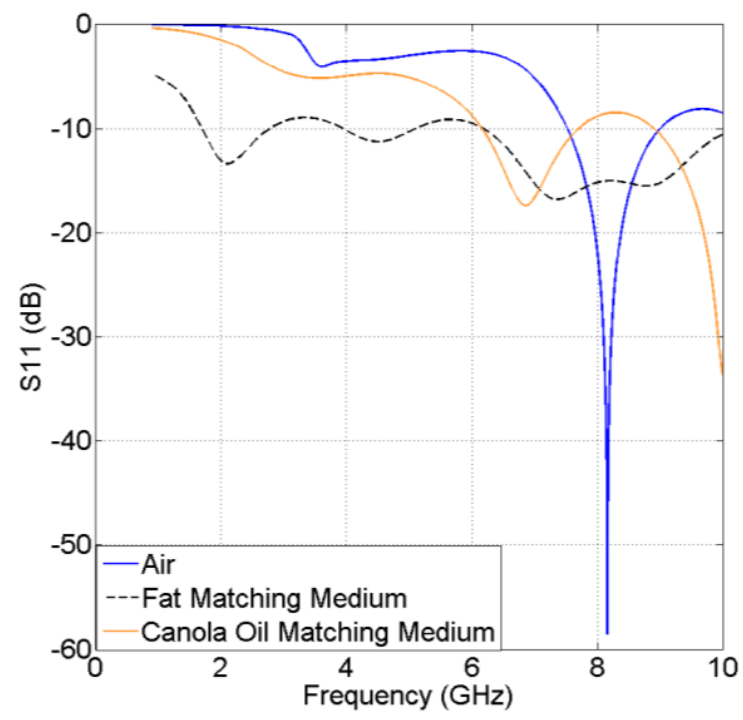

Fig. 2. Antenna performance in different mediums. This graph demonstrates the need for a matching medium to improve wave transmission. 
The tissues of the breast region are modelled in SEMCAD$\mathrm{X}$ as dispersive materials which behave in agreement with the single-pole Debye model (eq. (1)), where $\varepsilon(\omega)$ is the complex permittivity, $\varepsilon_{o}$ is the permittivity of free space, $\varepsilon_{\infty}$ is the dielectric constant of the material at infinite frequency, $\varepsilon_{s}$ is the static dielectric constant, $\sigma_{s}$ is the static conductivity, $\tau$ is the pole relaxation constant, and $\omega$ is the angular frequency:

$$
\varepsilon(\omega)=\varepsilon_{o}\left(\varepsilon_{\infty}+\frac{\sigma_{s}}{j \varepsilon_{0} \omega}+\frac{\varepsilon_{s}-\varepsilon_{\infty}}{1+j \omega \tau}\right)
$$

Table II lists the parameters that describe the Debye model for the skin layer, the chest wall, and the matching medium which are implemented within SEMCAD-X. The properties describing the tissues forming the interior of the breast region are described fully in [8].

TABLE II

Debye PARAmeters Describing DisPersive Model [5]

\begin{tabular}{|c|c|c|c|c|}
\hline & $\begin{array}{c}\text { Matching } \\
\text { Medium }\end{array}$ & Skin & Fat & Muscle \\
\hline$\varepsilon_{s}$ & 12.5 & 39.8 & 4.71 & 54.9 \\
\hline$\varepsilon_{\infty}$ & 9.0 & 15.9 & 3.12 & 21.7 \\
\hline$\sigma_{s}[\mathrm{~S} / \mathrm{m}]$ & 0.2 & 0.831 & 0.050 & 0.886 \\
\hline$\tau[\mathrm{psec}]$ & 69.0 & 13.0 & 13.0 & 27.8 \\
\hline
\end{tabular}

\section{Wave Excitation}

The simulations are conducted by exciting the antenna with a single frequency. The choice of frequency is based on the properties of several pulses. Specifically, we investigate three different pulses. The first pulse is a Gaussian modulated pulse with a centre frequency at $6.85 \mathrm{GHz}$, similar to the pulse investigated in [7]. The second pulse is a band-limited pulse restricted to the $2-4 \mathrm{GHz}$ range. This pulse is created experimentally with the use of a microstrip line [12]. We include this pulse for analysis since it represents an excitation waveform which we intend to investigate experimentally in the future. Results from this current analysis will shed light on the maximum power the pulse can carry before approaching current safety limits. The third pulse is created from a standard impulse generator, similar to the pulse used in the experimental system described in [11]. Fig. 3 graphs the time domain representation of each of the three pulses. Each of the three plots has been normalized to the peak amplitude of each pulse. Note that the Gaussian modulated pulse is far more time limited than both of the experimental pulses. Fig. 4 provides an illustration of the frequency content of each of the aforementioned pulses.

Unlike the analysis in [7], this paper focuses on SAR values as opposed to the specific absorption (SA) values. Due to the highly complex breast model and extremely fine mesh required to resolve the delicate structures of the antenna it becomes unfeasible to compute the SA values for pulse excitation. The SA computation would require a recording of the $E$ - and $H$ - fields at each voxel for every frequency of the pulse, something that would be impossible due to a limitation of computational resources. Hence, for this reason, we choose to evaluate the SAR values at specific frequencies. The frequencies chosen correspond to the centre frequency of each pulse as well as the two $-3 \mathrm{~dB}$ points (both high and low).

The entire simulation environment is shown in Fig. 5. This figure contains an illustration of the four possible antenna locations, Fig. 5(a), as well as the location of the various tissues comprising the breast model, Fig. 5(b). Odd and even antenna positions are cross-polarized; antennas in positions 1 and 3 have the same polarizations, whereas antennas in positions 2 and 4 are both cross-polarized (orientated with a 90-degree rotation in the $\mathrm{x}$-plane) with respect to the antennas in position 1 and 3 .
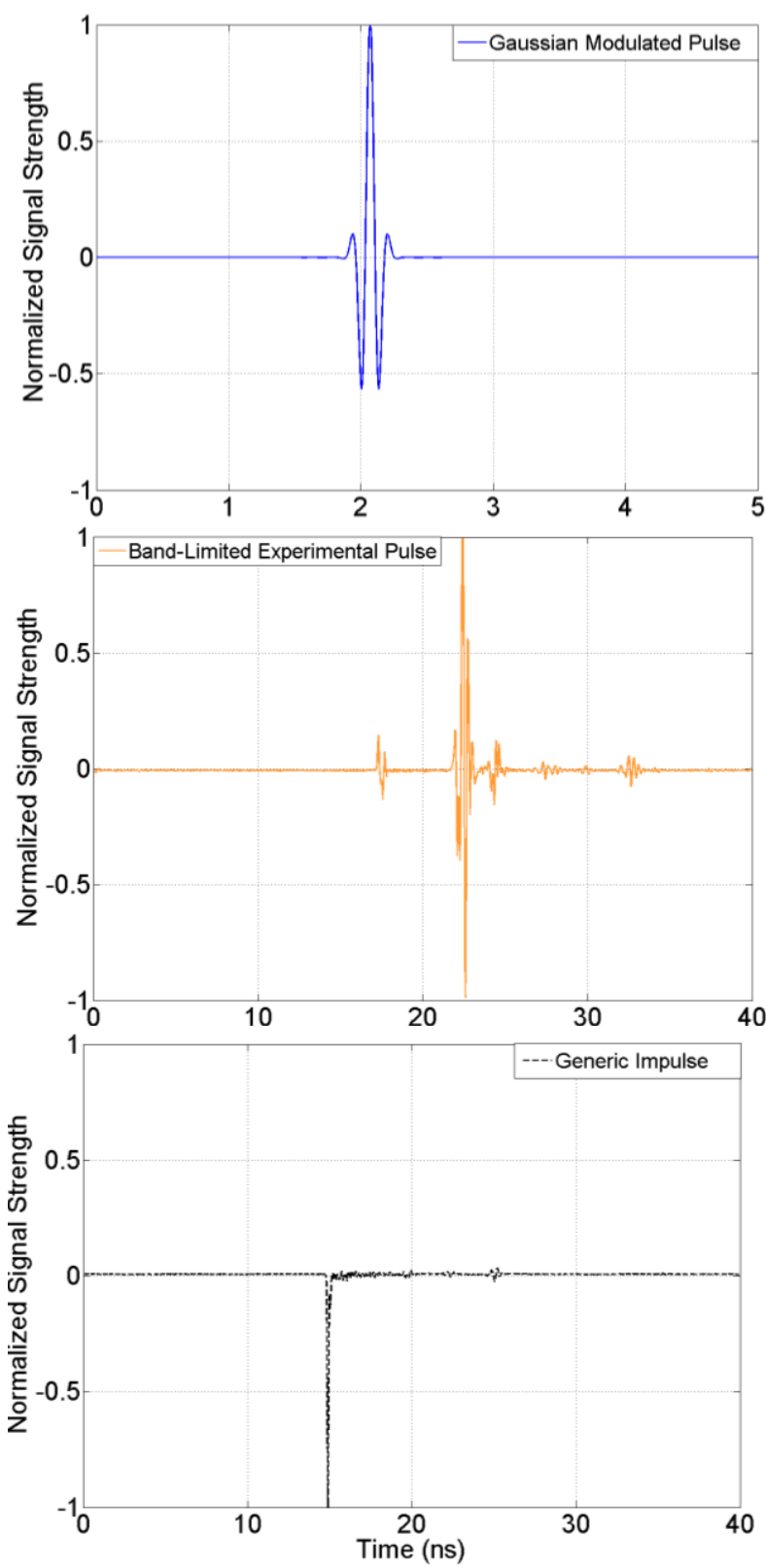

Fig. 3. Time domain representation of each of the three pulse shapes. The pulses have been normalized to the maximum amplitude of the individual pulses. Note that the Gaussian modulated pulse is on a much shorter timescale than the two experimental pulses. 


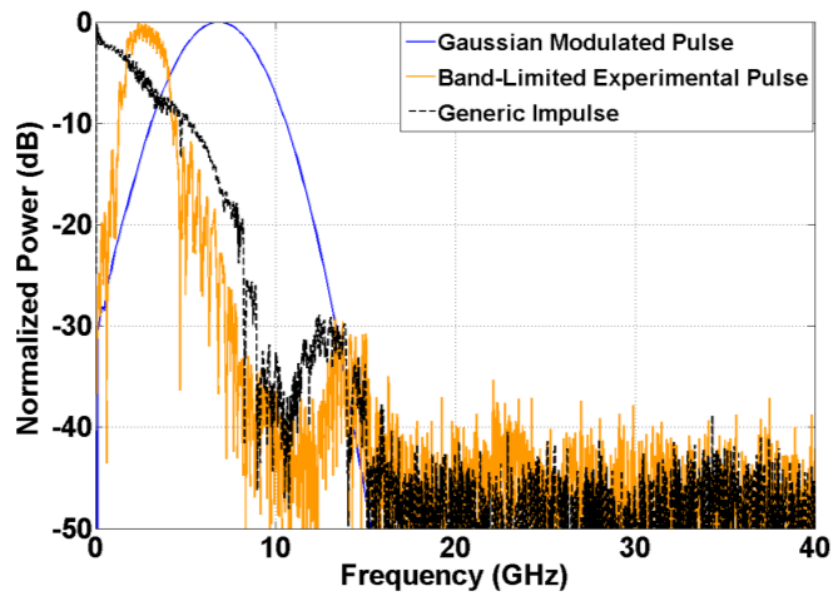

Fig. 4. A comparison of the frequency content of the three pulses investigated in the simulations.

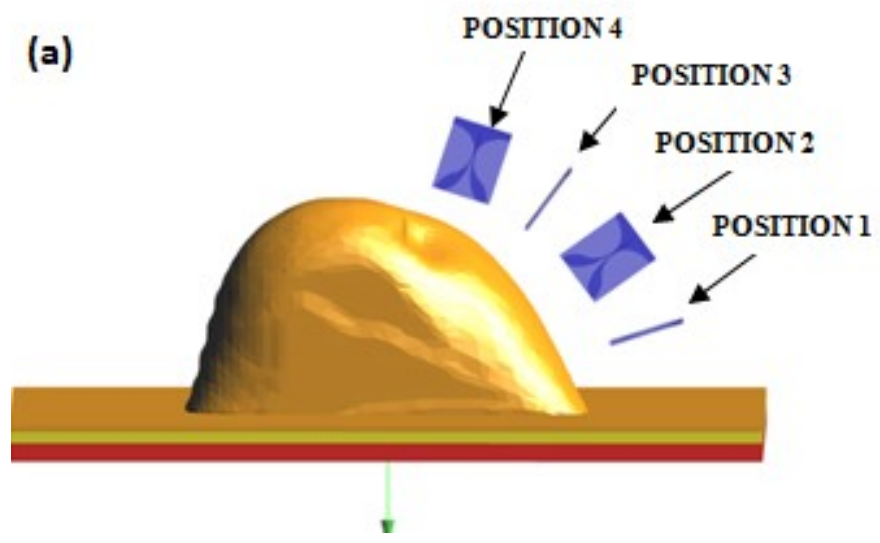

(b)

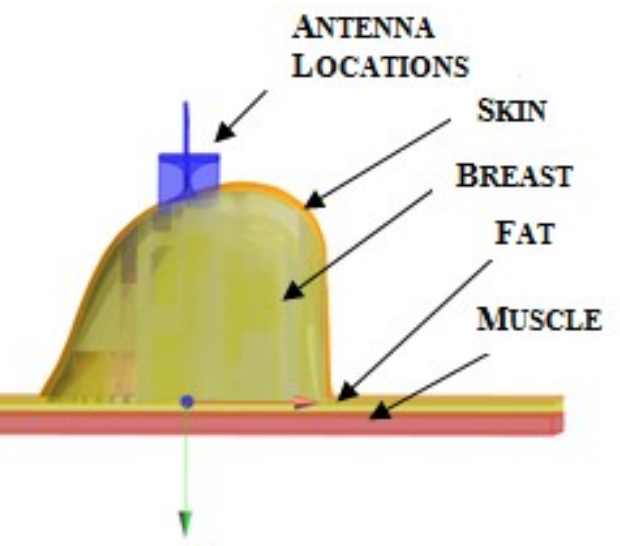

Fig. 5. A depiction of the complete simulation environment. (a) Side view denoting the four possible antenna locations. Note that the antennas in position 2 and 4 are cross-polarized in comparison to antennas placed in position 1 and 3. (b) Front view of the simulation environment denoting the layers of the breast model. Note that the breast is in front of the antenna from this viewing angle thereby partially hiding it, and that the antenna is at no point in contact with the skin.

\section{RESULTS}

The following section presents the data obtained from the FDTD simulations run within SEMCAD-X. We present the peak unaveraged SAR values, as well as the peak SAR values averaged over $10 \mathrm{~g}$ of tissue, from illuminating the breast with the three chosen frequencies (centre and both $-3 \mathrm{~dB}$ points), for each of the three pulses, at each of the four antenna positions. In all simulations the results have been normalized for $1 \mathrm{~mW}$ of incident power.

The peak unaveraged local SAR values represent the worstcase scenario. This metric can be used to pinpoint local hot spots within the tissue and to obtain a better understanding of the distribution of SAR values within the breast tissue. Fig. 6 presents the spatial distribution of the unaveraged SAR values. Fig. 6(a)-(d) each present an image formed by taking a crosssection through the breast tissue for each of the antenna positions 1 through 4 respectively (ex. Fig. 6(a) corresponds to the case when the breast is illuminated by the antenna in position 1). We note that these are not the same geometrical cross-sectional locations; rather, we choose to show, for each case, the cross-section that contains the maximum SAR point. In all the cases, both the images in Fig. 6 and those not presented within this paper, the peak unaveraged SAR values occur in the skin region closest to the antenna. This effect can clearly be seen in Fig. 6 as the bright spots move towards the nipple region as the antennas are shifted in the same direction. It is also interesting to note the occurrence of hot spots in the chest wall in the region below the antenna. As expected, regions in the breast furthest from the antenna experience the least amount of energy absorption.

Table III lists the maximum peak unaveraged local SAR value computed across all frequencies for each of the antenna positions. For each of the four antenna positions, the excitation of the antenna with the highest frequency, $8.9 \mathrm{GHz}$, resulted in the maximum energy absorption. This trend is not surprising and confirms the suspicions about the relationship between frequency and peak local SAR values presented in [5]. The maximum energy absorption in our study occurs when the antenna is located in position 4.

TABLE III

PEAK UNAVERAGED SAR VALUES FOR SPECIFIC ANTENNA POSITION AND CORRESPONDING FREQUENCY OF EXCITATION

\begin{tabular}{|c|c|c|}
\hline $\begin{array}{c}\text { Antenna } \\
\text { Position }\end{array}$ & $\begin{array}{c}\text { Peak Unaveraged SAR } \\
\text { [mW/kg] }\end{array}$ & $\begin{array}{c}\text { Frequency } \\
\text { [GHz] }\end{array}$ \\
\hline Position 1 & 177 & 8.9 \\
\hline Position 2 & 171 & 8.9 \\
\hline Position 3 & 248 & 8.9 \\
\hline Position 4 & 643 & 8.9 \\
\hline
\end{tabular}




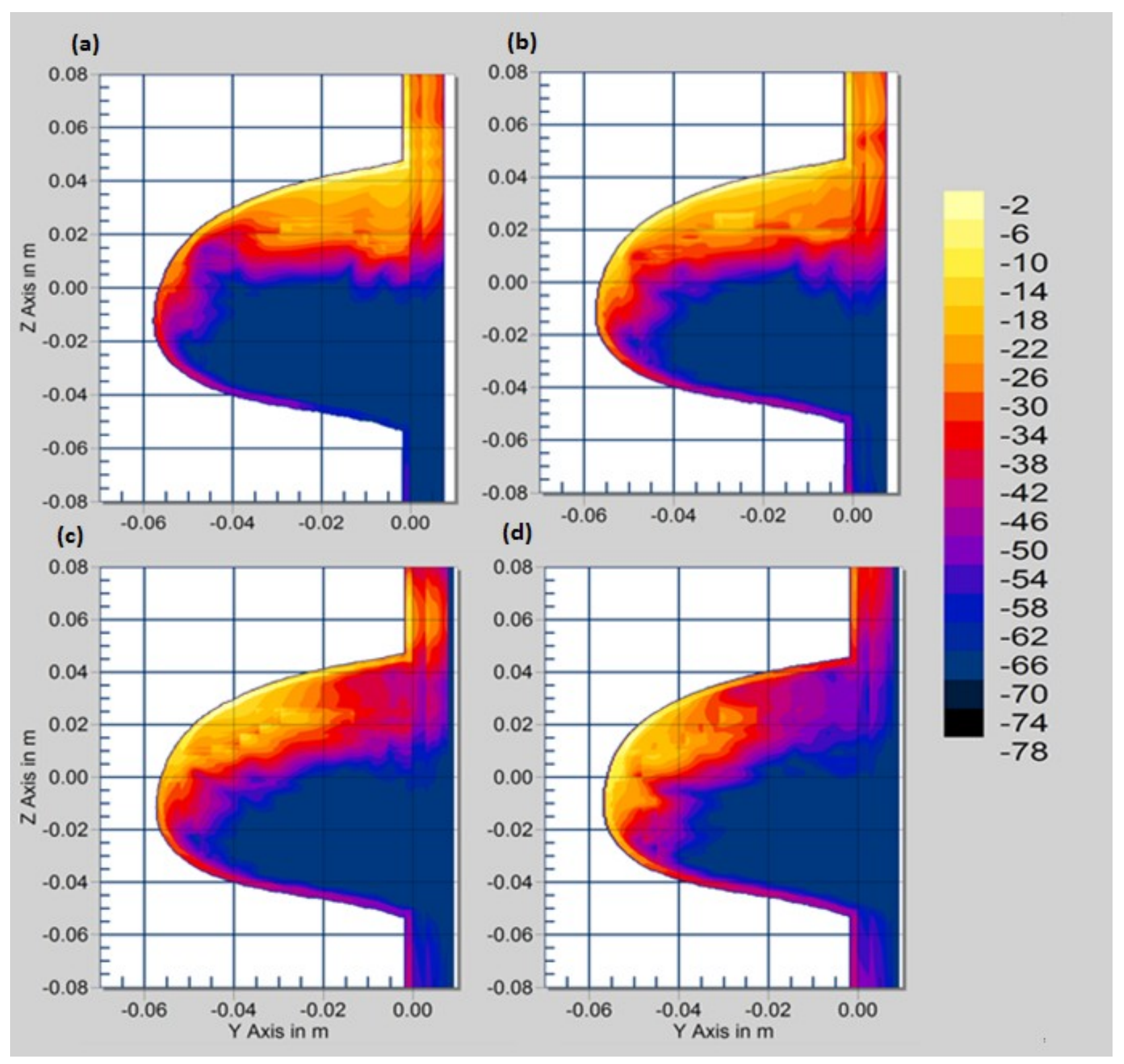

Fig. 6. An example of the distribution of unaveraged SAR values through different cross-sections of the breast. (a)-(d) Show the distribution of unaveraged SAR values for illumination of the breast at $8.9 \mathrm{GHz}$ with the antenna located in positions 1 through 4 respectively, as depicted in Fig. 5(a). Each cross-section contains the peak unaveraged SAR value for each respective scenario. The scale is noted in dB relative to this local peak SAR value. Note how the antenna position changes the location of the local peak as well as the distribution of the absorbed energy within the breast.

Table IV presents the data obtained when computing the peak 10 -g averaged SAR values. For an input power of $1 \mathrm{~mW}$ we note that the maximum recorded 10 -g averaged SAR value, $10.0 \mathrm{~mW} / \mathrm{kg}$, is well below, by approximately a factor of 400 , the limits established in Table I. Similar to the observation noted for the peak unaveraged SAR values, for each of the four antenna positions the maximum 10-g averaged SAR values occur for the highest excitation frequency.

We also note that the 10 -g average values, much like the peak unaveraged SAR values, are higher for the antennas located in positions 3 and 4 . This is possibly due to the distribution of the breast tissue components. A high portion of glandular tissue is located near the nipple region of the breast. The high conductivity of the glandular tissue may cause additional energy to be absorbed when compared to the surrounding fatty tissue, which has a much lower conductivity. Since the glandular tissue is located near the skin surface it causes the average SAR values near the nipple region to be higher. Therefore, when the antenna is in positions 3 and 4 , and the peak SAR values are observed on the skin surface near the nipple region, we can expect the $10 \mathrm{~g}$ average to be increased when compared to positions 1 and 2 when the peak SAR values are on the breast surface closer to the chest wall. 
TABLE IV

PeAK 10-g SAR VALUeS FOR SPECIFIC ANTENNA Position AND CORRESPONDING FREQUENCY OF EXCITATION

\begin{tabular}{|c|c|c|}
\hline $\begin{array}{c}\text { Antenna } \\
\text { Position }\end{array}$ & $\begin{array}{c}\text { Peak 10-g SAR } \\
{[\mathbf{m W} / \mathbf{k g}]}\end{array}$ & $\begin{array}{c}\text { Frequency } \\
{[\mathbf{G H z}]}\end{array}$ \\
\hline Position 1 & 4.66 & 8.9 \\
\hline Position 2 & 4.64 & 8.9 \\
\hline Position 3 & 6.02 & 8.9 \\
\hline Position 4 & 10.0 & 8.9 \\
\hline
\end{tabular}

\section{CONCLUSION}

Based on the peak 10-g averaged SAR values we have demonstrated that the energy absorbed by the breast, when excited by an antenna at various locations in the near field, and over several select frequencies, falls below the established safety values for $1 \mathrm{~mW}$ of incident power. We have also noted the effect that the varying antenna positions have on the distribution of peak unaveraged SAR values and reaffirmed the relationship between increasing frequency and increasing local energy absorption. Furthermore, we demonstrated that the peak unaveraged SAR value, regardless of antenna position and excitation frequency, always occurs in the skin region nearest to the illuminating antenna.

In this work, we have illuminated the breast structure with an antenna that is currently used in an experimental imaging system and excite the antenna with the frequencies carrying the most power in the experimental pulses employed for detection. Since the data in this analysis have been normalized to $1 \mathrm{~mW}$ of incident power it is possible to extrapolate the maximum amount of power that can be sent in order to adhere to the safety limits in place. The results of this study can provide guidance for the maximum amount of power these pulses can carry in future experimental work.

\section{ACKNOWLEDGMENT}

The authors are grateful for the funding support by the Natural Sciences and Engineering Research Council of Canada (NSERC), le Fonds québécois de la recherche sur la nature et les technologies (FQRNT), and Partenariat de Recherche Orientée en Microélectronique, Photonique et Télécommunications (PROMPT).

\section{REFERENCES}

[1] Canadian Cancer Society. (2010, August 17). What is breast cancer? [Online]. Available: http://www.cancer.ca/

[2] E.C. Fear, P.M. Meaney and M.A. Stuchly "Microwaves for breas cancer detection?" IEEE Potentials, pp.12-18, February/March 2003

[3] Health Canada, "Limits of Human Exposure to Radiofrequency Electromagnetic Fields in the Frequency Range from $3 \mathrm{kHz}$ to 300 GHz: Safety Code 6 ," Health Canada, 2009.

[4] M. Lazebnik, D. Popovic, L. McCartney, C. Watkins, M. Lindstrom, J. Harter, S. Sewall, T. Ogilvie, A. Magliocco, T. Breslin, W. Temple, D. Mew, J. Booske, M. Okoniewski, and S. Hagness, "A large-scale study of the ultrawideband microwave dielectric properties of normal, benign and malignant breast tissues obtained from cancer surgeries," Phys. Med. Biol., Vol. 52, pp. 6093-6115, 2007.

[5] A. Santorelli, M.V. Schueren and M. Popović, "SAR Levels in Microwave Breast Imaging: 3-D Safety Assessment with Plane-Wave
Illumination," Accepted at The Asia Pacific Microwave Conference (APMC 2011), Melbourne, Australia, December 5-8, 2011.

[6] P. Bernardi, M. Cavagnaro, S. Pisa, E. Piuzzi, "Specific Absorption Rate and Temperature Elevation in a Subject Exposed in the Far-Field of Radio-Frequency Sources operating in the 10-900-Mhz Range," IEEE Trans. Biomed. Eng., vol. 50, pp. 295-304, March 2003.

[7] E. Zastrow, S. Davis, M. Lazebnik, F. Kelcz, B. V. Veen, and S Hagness, "Development of anatomically realistic numerical breas phantoms with accurate dielectric properties for modeling microwave interactions with the human breast," IEEE Trans. Biomed. Eng., vol. 55, no. 12 , pp. 2792-2800, Dec. 2008 .

[8] G. Zhu, B. Oreshkin, E. Porter, M. Coates, M. Popović "Numerical Breast Models for Commercial FDTD Simulators", in Proc. European Conference on Antennas and Propagation (Eu-CAP'09), Berlin, Germany, Mar. 2009

[9] SPEAG, Schmid \& Partner Engineering AG. (2011, May 3) [Online].Available: http://www.speag.com/

[10] H. Kanj and M. Popović, "A Novel Ultra-Compact Broadband Antenna for Microwave Breast Tumor Detection,." Progress in Electromagnetics Research, vol. 86, pp. 169-198, 2008.

[11] E. Porter, A. Santorelli, M. Coates, and M. Popović, "An Experimental System for Time-Domain Microwave Breast Imaging," in Proc. 5th European Conference on Antennas and Propagation (EUCAP 2011), Rome, Italy, April 11-15, 2011.

[12] I. Arnedo, J.D. Schwartz, M.A.G. Laso, T. Lopetegi, D.V. Plant, J. Azaa, "Passive Microwave Planar Circuitsfor Arbitrary UWB Pulse Shaping," Microwave and Wireless Components Letters, IEEE, vol.18, no.7, pp.452-454, July 2008 